\title{
New Exact Solutions for MHD Transient Rotating Flow of a Second-Grade Fluid in a Porous Medium
}

\author{
Faisal Salah, Zainal Abdul Aziz, and Dennis Ling Chuan Ching \\ Department of Mathematics, Faculty of Science, Universiti Teknologi Malaysia, 81310 UTM Skudai, \\ Johor, Malaysia \\ Correspondence should be addressed to Zainal Abdul Aziz, abdulazizzainal@gmail.com
}

Received 22 October 2010; Revised 17 February 2011; Accepted 19 March 2011

Academic Editor: V. Kumaran

Copyright (C) 2011 Faisal Salah et al. This is an open access article distributed under the Creative Commons Attribution License, which permits unrestricted use, distribution, and reproduction in any medium, provided the original work is properly cited.

The magnetohydrodynamic (MHD) and rotating flow of second-grade fluid over a suddenly moved flat plate is investigated, where the second-grade fluid saturates the porous medium. The new exact solution is derived by using the Fourier sine and Laplace transforms. Many interesting available results in the literature are obtained as limiting cases of our solution. Finally, some graphical results are presented for different values of the material constants.

\section{Introduction}

The study of non-Newtonian fluids in a porous medium and rotating frame offers special challenges to mathematicians, numerical analysts, and engineers. Some of these studies are notable and applied in paper, food stuff, personal care product, textile coating, and suspension solutions industries. The non-Newtonian fluids have been mainly classified under the differential, rate, and integrals types. The second-grade fluids are the subclass of non-Newtonian fluids and are the simplest subclass of differential type fluids which can show the normal stress effects. It was employed to study various problems due to their relatively simple structure. Moreover, one can reasonably hope to obtain exact solutions from this type of second-grade fluid. This motivates us to choose the second-grade model in this study. The exact solutions are important, as these provide standard for checking the accuracies of many approximate solutions which can be numerical or empirical. They can also be used as tests for verifying numerical schemes that are developed for studying more complex flow problems.

In general, the governing equation in second-grade fluid is of third order, which is higher than the second-order Navier-Stokes equation. Furthermore, the order of equation in second-grade fluid is reduced when higher order nonlinearities are ignored, which is not 
possible in the Navier-Stokes equations. Exact solution of the problem is given by using the Fourier sine and Laplace transforms method. This method has already been successfully applied by various workers, for example, Fetecau et al. [1,2]. Justifiably, the traditional Fourier sine and Laplace transforms method has the following important features. It is a very powerful technique for solving these kinds of problems, which literally transforms the original linear differential equation into an elementary algebraic expression. More importantly, the transformation avoids the omission of a critical term from the resulted subsidiary equation.

The analysis of the effects of rotation and magnetohydrodynamic (MHD) flows through a porous medium has gained an increasing interest due to the wide range of applications either in geophysics or in engineering such as the optimization of the solidification process of metals and metal alloys and the control underground spreading of chemical wastes and pollutants. MHD is the study of the interaction of conducting fluids with electromagnetic phenomena. The flow of an electrically conducting fluid in the presence of magnetic field is of importance in various areas of technology and engineering such as MHD power generation and MHD pumps. Therefore, several researchers have discussed the flows of second-grade fluid in different configurations, and there are on hand few attempts which include the effects of rotation and MHD (for instances, see studies in [3-27] and references therein).

The objective of the current study is to establish new exact solutions for the velocity field corresponding to the first problem of Stokes for a second-grade fluid. The fluid is magnetohydrodynamic (MHD) in the presence of an applied magnetic field, and it occupies a half porous space, which is bounded by a rigid and nonconducting plate, and the whole system is also rotating.

\section{Formulation of the Problem}

Let us consider a Cartesian coordinate system $(x, y, z)$. We consider a fluid saturated porous half space bounded by an infinite accelerated plate at $z=0$ ( $z$-axis is taken normal to the plate). The whole system is rotating uniformly with a constant angular velocity $\Omega$ about the $z$-axis. The porous space is described by the modified Darcy's law. A constant magnetic field $B_{\circ}$ acts in the $z$-direction. The applied and induced magnetic fields are chosen zero. The equations governing the present flow are [21]

$$
\begin{aligned}
& \rho\left(\frac{\partial u}{\partial t}-2 \Omega v\right)=\mu \frac{\partial^{2} u}{\partial z^{2}}+\alpha_{1} \frac{\partial^{3} u}{\partial z^{2} \partial t}-\sigma B_{\circ}^{2} u-\frac{\mu \varphi}{k}\left(1+\frac{\alpha_{1}}{\mu} \frac{\partial}{\partial t}\right) u, \\
& \rho\left(\frac{\partial v}{\partial t}+2 \Omega u\right)=\mu \frac{\partial^{2} v}{\partial z^{2}}+\alpha_{1} \frac{\partial^{3} v}{\partial z^{2} \partial t}-\sigma B_{\circ}^{2} v-\frac{\mu \varphi}{k}\left(1+\frac{\alpha_{1}}{\mu} \frac{\partial}{\partial t}\right) v .
\end{aligned}
$$

In above equations $\rho, t, \mu, \sigma, \alpha_{1}, \varphi$, and $k$, respectively, indicate the fluid density, time, dynamic viscosity, electrical conductivity, material parameter of second-grade fluid, porosity, and the permeability of porous medium. 
The initial and boundary conditions are

$$
\begin{gathered}
u=v=0 \quad \text { when } t=0, z>0 \\
u(0, t)=U_{\circ}, \quad v(0, t)=0 \text { for } t>0 \\
u, \frac{\partial u}{\partial z}, v, \frac{\partial v}{\partial z} \rightarrow 0 \quad \text { as } z \rightarrow \infty, t>0 .
\end{gathered}
$$

\section{Solution of the Problem}

Defining $F=u+i v$

(2.1) can be combined as

$$
\frac{\partial F}{\partial t}+\left(2 i \Omega+\frac{\sigma B_{\circ}^{2}}{\rho}\right) F=v \frac{\partial^{2} F}{\partial z^{2}}+\frac{\alpha_{1}}{\rho} \frac{\partial^{3} F}{\partial z^{2} \partial t}-\frac{v \varphi}{k}\left(1+\frac{\alpha_{1}}{\mu} \frac{\partial}{\partial t}\right) F,
$$

where $v$ is the kinematic viscosity. The appropriate boundary and initial conditions are $F(0, t)=U_{\circ}, t>0 ; F(z, 0)=0, z>0$,

$$
F(z, t), \frac{\partial F(z, t)}{\partial z} \rightarrow 0 \quad \text { as } z \rightarrow \infty, t>0
$$

In order to solve the linear partial differential equation (3.1) with initial and boundary conditions (3.2), we will use the Fourier sine and Laplace transforms. For a greater generality, we consider the boundary condition $F(0, t)=U_{\circ}(t)$ with $U_{\circ}(0)=0$ and apply the Fourier sine transform with respect to. We then obtain

$$
\frac{\partial F_{s}(\eta, t)}{\partial t}+\frac{\left[v \eta^{2}+v(\varphi / k)+c\right]}{\left[1+\alpha \eta^{2}+\alpha(\varphi / k)\right]} F_{s}(\eta, t)=\frac{\eta \sqrt{2 / \pi}\left[v U_{\circ}(t)+\alpha U_{\circ}^{\prime}(t)\right]}{\left(1+\alpha \eta^{2}+\alpha(\varphi / k)\right)}, \quad t>0,
$$

where $\alpha=\alpha_{1} / \rho, c=2 i \Omega+\sigma B_{\circ}^{2} / \rho$, and the Fourier sine transform $F_{s}(\eta, t)$ of $F(z, t)$ has to satisfy the conditions

$$
F_{S}(\eta, 0)=0, \quad \eta>0
$$

Applying the Laplace transform to (3.3) and using the initial condition (3.4), we find that

$$
\bar{F}_{s}(\eta, q)=\frac{\eta \sqrt{2 / \pi}[v+\alpha q] \bar{U}_{\circ}(q)}{\left(v \eta^{2}+v(\varphi / k)+c\right)+q\left(1+\alpha \eta^{2}+\alpha(\varphi / k)\right)}
$$

where $q$ is the transform parameter, while $\bar{F}_{s}(\eta, q)$ and $\bar{U}_{\circ}(q)$ are the Laplace transform of $F_{s}(\eta, t)$ and $U_{\circ}(t)$, respectively. Choosing $U_{\circ}(t)=U H(t)$, where $H(t)$ is Heaviside unit step function and $U$ is the constant, we get the velocity field corresponding to the first problem of Stokes. 
In the case $\bar{U}_{\circ}(q)=U / q,(3.5)$ takes the form

$$
\bar{F}_{s}(\eta, q)=\frac{\eta \sqrt{2 / \pi} U[v+\alpha q]}{q^{2}\left(v \eta^{2}+v(\varphi / k)+c\right)+q\left(1+\alpha \eta^{2}+\alpha(\varphi / k)\right)} .
$$

Applying the inverse Laplace transform to (3.6), the solution can be expressed as

$$
\begin{aligned}
F_{S}(\eta, t)=\eta \sqrt{\frac{2}{\pi}} U\left[\frac{v\left(1-e^{-\left(v \eta^{2}+v(\varphi / k)+c\right) t /\left(1+\alpha \eta^{2}+\alpha(\varphi / k)\right)}\right)}{\left(v \eta^{2}+v(\varphi / k)+c\right)}\right. \\
\\
\left.\quad+\frac{\alpha}{\left(1+\alpha \eta^{2}+\alpha(\varphi / k)\right)} e^{-\left(v \eta^{2}+v(\varphi / k)+c\right) t /\left(1+\alpha \eta^{2}+\alpha(\varphi / k)\right)}\right], \quad t>0 .
\end{aligned}
$$

Inversion of Fourier sine transform in (3.7) gives

$$
\begin{gathered}
F(z, t)=U H(t)\left[e^{-(\sqrt{\varphi / k+c / v}) z}-\frac{2}{\pi} \int_{0}^{\infty} \frac{\eta e^{-\left(\nu \eta^{2}+v(\varphi / k)+c\right) t /\left(1+\alpha \eta^{2}+\alpha(\varphi / k)\right)}}{\left(\eta^{2}+\varphi / k+c / v\right)} \sin (z \eta) d \eta\right. \\
\left.+\frac{2}{\pi} \int_{0}^{\infty} \frac{\eta e^{-\left(v \eta^{2}+v(\varphi / k)+c\right) t /\left(1+\alpha \eta^{2}+\alpha(\varphi / k)\right)}}{\left(\eta^{2}+\varphi / k+1 / \alpha\right)} \sin (z \eta) d \eta\right] .
\end{gathered}
$$

Expression (3.8) is the new exact solution for the velocity field corresponding to the first problem of Stokes for a second-grade fluid, which is rotating and magnetohydrodynamic. The above expression for hydrodynamic fluid $\left(B_{\circ}^{2}=0\right)$ in a nonporous space $(\varphi=0)$ is given by

$$
\begin{gathered}
F(z, t)=U H(t)\left[e^{-(1+i) \sqrt{\Omega / v} z}-\frac{2 v}{\pi} \int_{0}^{\infty} \frac{\eta e^{-\left(v \eta^{2}+2 i \Omega\right) t /\left(1+\alpha \eta^{2}\right)}}{\left(v \eta^{2}+2 i \Omega\right)} \sin (z \eta) d \eta\right. \\
\left.+\frac{2 \alpha}{\pi} \int_{0}^{\infty} \frac{\eta e^{-\left(v \eta^{2}+2 i \Omega\right) t /\left(1+\alpha \eta^{2}\right)}}{\left(\alpha \eta^{2}+1\right)} \sin (z \eta) d \eta\right]
\end{gathered}
$$

Making $(\Omega=0)$ into (3.9), we obtain

$$
F(z, t)=U H(t)\left[1-\frac{2}{\pi} \int_{0}^{\infty} \frac{\sin (z \eta)}{\eta\left(1+\alpha \eta^{2}\right)} \exp \left(-\frac{v \eta^{2} t}{1+\alpha \eta^{2}}\right) d \eta\right]
$$

The velocity field $F(z, t)$, given by (3.10), has been recently obtained by Fetecau et al. [1, Equation (16)]. 


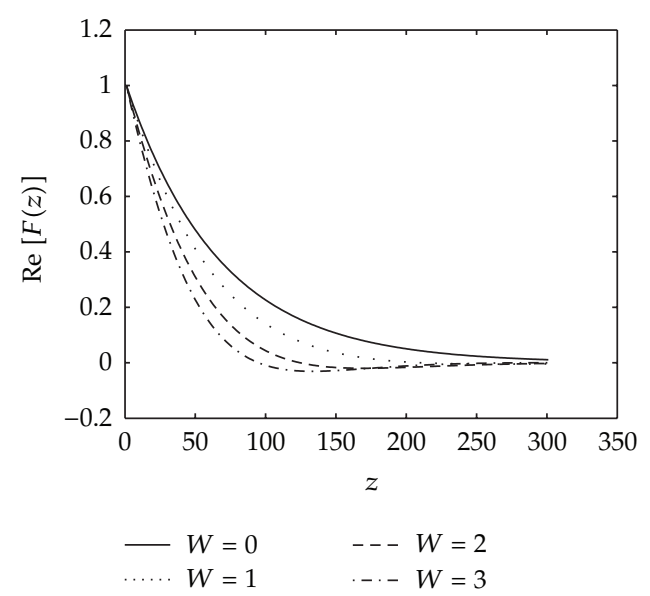

(a)

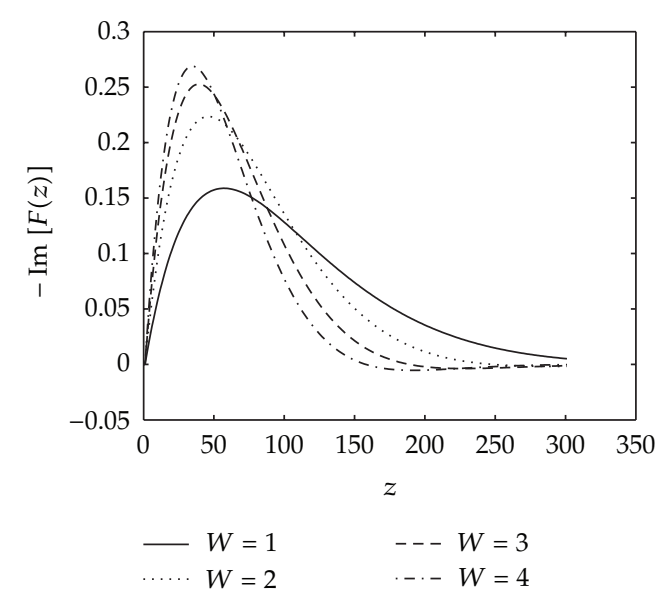

(b)

Figure 1: Profiles of the normalized steady state velocity $F(z)$ for various values of $W$. (a) Shows that the real part of the velocity profile decreases for various values of rotation $W$, with respect to the increase in $z$. As each $W$ increases, the corresponding velocity profile decreases. (b) Indicates that the magnitude of imaginary part of each of the velocity profile increases initially and later reduces for various corresponding values of rotation $W$, with respect to the increase in $z$. Similar result is obtained in Hayat et al. [21].

Result (3.8) for a magnetohydrodynamic viscous fluid $(\alpha=0)$ in a porous space is

$$
F(z, t)=U H(t)\left[e^{-(\sqrt{\varphi / k+c / v}) z}-\frac{2}{\pi} \int_{0}^{\infty} \frac{\eta e^{-\left(v \eta^{2}+v(\varphi / k)+c\right) t}}{\left(\eta^{2}+\varphi / k+c / v\right)} \sin (z \eta) d \eta\right]
$$

and the steady solution is

$$
F(z)=U e^{-(\sqrt{\varphi / k+c / v}) z} .
$$

\section{Results and discussions}

In this section, we present the graphical illustrations of the velocity profiles which have been determined for the flow due to the impulsive motion of an infinite flat plate. The emerging parameters are defined here as

(i) $W=\Omega$ is the rotating parameter,

(ii) $M=\sigma B_{\circ}^{2} / \rho$ is the magnetic field parameter,

(iii) $k$ is the permeability of the porous medium.

In order to illustrate the role of these parameters on the real and imaginary parts of the velocity $F$, the Figures 1-3 have been displayed. In these Figures, panels (a) depict the variations of $\operatorname{Re}[F(z)]$, and panels $(b)$ indicate the variations of $-\operatorname{Im}[F(z)]$. 


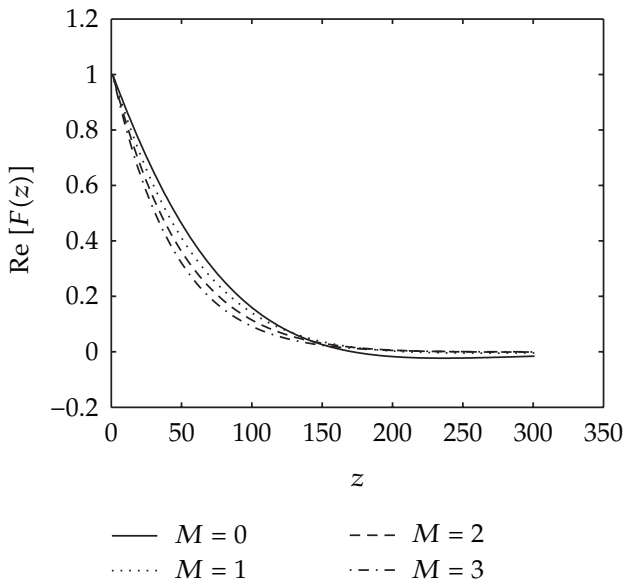

(a)

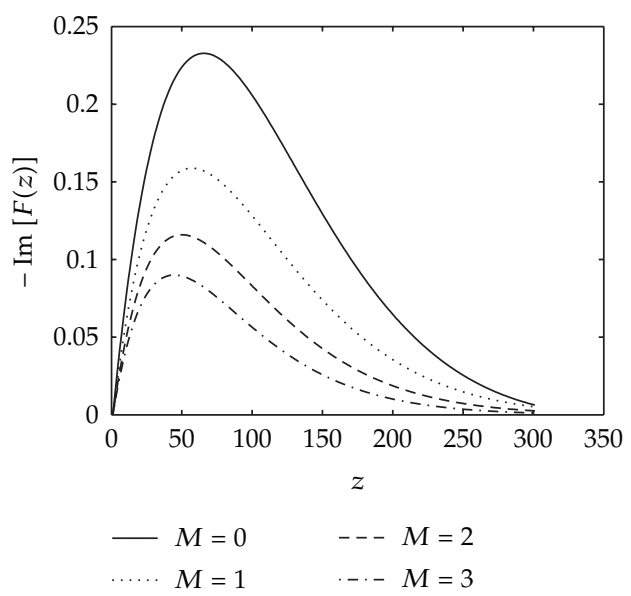

(b)

Figure 2: Profiles of the normalized steady state velocity $F(z)$ for various values of $M$. (a) Points to the effects of MHD parameter $M$, in the real part of velocity profile. By increasing each parameter $M$, it is noted that the corresponding real part of the velocity profile decreases. (b) Also denotes the effects of magnetic field on the imaginary part of the velocity profile. By increasing each of $M$, it is noted that the corresponding imaginary part decreases initially and later increases.

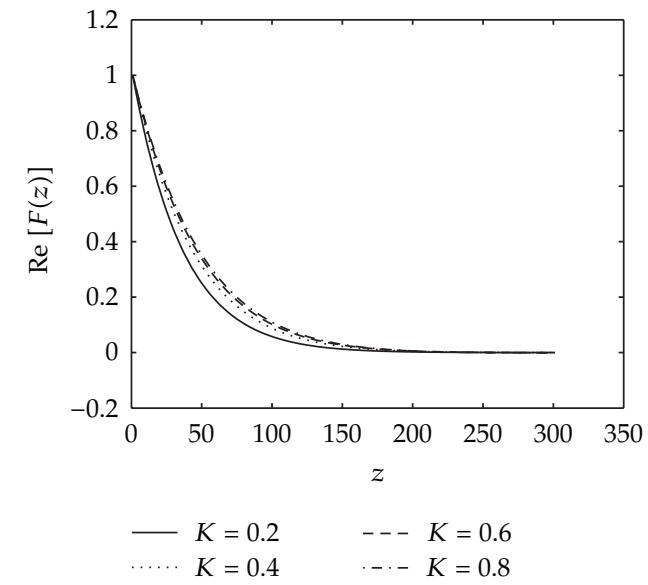

(a)

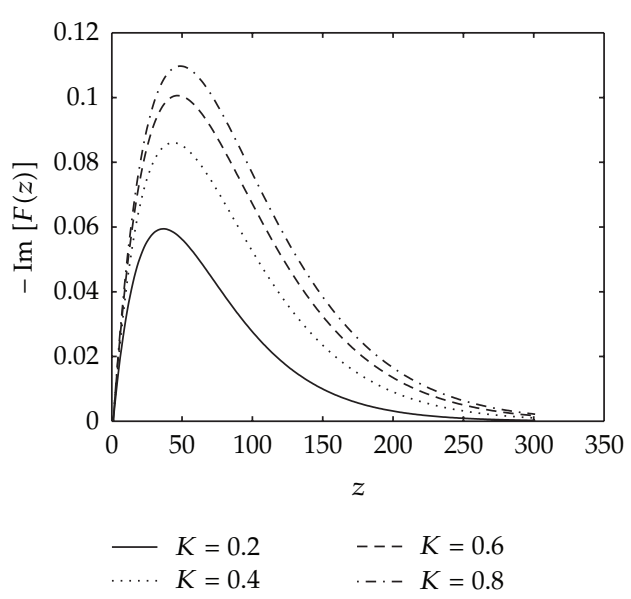

(b)

Figure 3: Profiles of the normalized steady state velocity $F(z)$ for various values of $K$. (a) Shows that with an increase in each of the porosity parameter $k$, the corresponding real part of the velocity field decreases. (b) Illustrates that by increasing each of the porosity parameter $k$, the magnitude of the corresponding imaginary part of velocity field increases initially and later slows down.

\section{Conclusion}

Equation (3.3) and the ensuing results attained above are new extensions of those obtained in other comparable existing study in Hayat et al. [21]. For example, if we were to choose $U_{\circ}(t)=U \sin (\omega t)$ or $U_{\circ}(t)=U t$ in (3.3), then the solutions corresponding to the second problem of Stokes or the flow due to a constant accelerated plate can be recovered. 


\section{Acknowledgments}

F. Salah is thankful to the Sudanese government for financial support. D. L. C. Ching is grateful to MOSTI for the NSF scholarship. This research is partially funded by MOHE FRGS Vote nos. 78485 and 78675 . The authors wish to thank the anonymous reviewers whose comments led to some improvements of the presentation of the results.

\section{References}

[1] C. Fetecau, T. Hayat, M. Khan, and C. Fetecau, "Erratum: unsteady flow of an Oldroyd-B fluid induced by the impulsive motion of a plate between two side walls perpendicular to the plate," Acta Mechanica, vol. 216, no. 1-4, pp. 359-361, 2011.

[2] C. Fetecau, T. Hayat, J. Zierep, and M. Sajid, "Energetic balance for the Rayleigh-Stokes problem of an Oldroyd-B fluid," Nonlinear Analysis: Real World Applications, vol. 12, no. 1, pp. 1-13, 2011.

[3] F. Shen, W. Tan, Y. Zhao, and T. Masuoka, "The Rayleigh-Stokes problem for a heated generalized second grade fluid with fractional derivative model," Nonlinear Analysis, vol. 7, no. 5, pp. 1072-1080, 2006.

[4] W. C. Tan and T. Masuoka, "Stokes' first problem for a second grade fluid in a porous half-space with heated boundary," International Journal of Non-Linear Mechanics, vol. 40, no. 4, pp. 515-522, 2005.

[5] K. R. Rajagopal and A. S. Gupta, "On a class of exact solutions to the equations of motion of a second grade fluid," International Journal of Engineering Science, vol. 19, no. 7, pp. 1009-1014, 1981.

[6] R. Bandelli and K. R. Rajagopal, "Start-up flows of second grade fluids in domains with one finite dimension," International Journal of Non-Linear Mechanics, vol. 30, no. 6, pp. 817-839, 1995.

[7] M. E. Erdogan and C. E. Imrak, "On unsteady unidirectional flows of a second grade fluid," International Journal of Non-Linear Mechanics, vol. 40, no. 10, pp. 1238-1251, 2005.

[8] C. Fetecau, T. Hayat, C. Fetecau, and N. Ali, "Unsteady flow of a second grade fluid between two side walls perpendicular to a plate," Nonlinear Analysis: Real World Applications, vol. 9, no. 3, pp. 1236-1252, 2008.

[9] M. Yürüsoy, M. Pakdemirli, and Ö. F. Noyan, "Lie group analysis of creeping flow of a second grade fluid," International Journal of Non-Linear Mechanics, vol. 36, no. 6, pp. 955-960, 2001.

[10] P. D. Ariel, "A numerical algorithm for computing the stagnation point flow of a second grade fluid with/without suction," Journal of Computational and Applied Mathematics, vol. 59, no. 1, pp. 9-24, 1995.

[11] C. Xue and J. Nie, "Exact solutions of the Rayleigh-Stokes problem for a heated generalized second grade fluid in a porous half-space," Applied Mathematical Modelling, vol. 33, no. 1, pp. 524-531, 2009.

[12] J. Zierep and C. Fetecau, "Energetic balance for the Rayleigh-Stokes problem of a second grade fluid," International Journal of Engineering Science, vol. 45, no. 1, pp. 155-162, 2007.

[13] T. Hayat, R. Ellahi, S. Asghar, and A. M. Siddiqui, "Flow induced by non-coaxial rotation of a porous disk executing non-torsional oscillations and a second grade fluid rotating at infinity," Applied Mathematical Modelling, vol. 28, no. 6, pp. 591-605, 2004.

[14] M. Hussain, T. Hayat, S. Asghar, and C. Fetecau, "Oscillatory flows of second grade fluid in a porous space," Nonlinear Analysis: Real World Applications, vol. 11, no. 4, pp. 2403-2414, 2010.

[15] M. Khan, E. Naheed, C. Fetecau, and T. Hayat, "Exact solutions of starting flows for second grade fluid in a porous medium," International Journal of Non-Linear Mechanics, vol. 43, no. 9, pp. 868-879, 2008.

[16] S. Asghar, T. Hayat, and P. D. Ariel, "Unsteady Couette flows in a second grade fluid with variable material properties," Communications in Nonlinear Science and Numerical Simulation, vol. 14, no. 1, pp. 154-159, 2009.

[17] T. Hayat, S. Nadeem, R. Ellahi, and S. Asghar, "The influence of Hall current in a circular duct," Nonlinear Analysis: Real World Applications, vol. 11, no. 1, pp. 184-189, 2010.

[18] P. Puri, "Rotary flow of an elastico-viscous fluid on an oscillating plate," Zeitschrift für Angewandte Mathematik und Mechanik, vol. 54, no. 11, pp. 743-745, 1974.

[19] P. Puri and P. K. Kulshrestha, "Rotating flow of non-Newtonian fluids," Applicable Analysis, vol. 4, pp. 131-140, 1974.

[20] T. Hayat, K. Hutter, S. Asghar, and A. M. Siddiqui, "MHD flows of an Oldroyd-B fluid," Mathematical and Computer Modelling, vol. 36, no. 9-10, pp. 987-995, 2002. 
[21] T. Hayat, C. Fetecau, and M. Sajid, "Analytic solution for MHD transient rotating flow of a second grade fluid in a porous space," Nonlinear Analysis: Real World Applications, vol. 9, no. 4, pp. 1619-1627, 2008.

[22] T. Hayat, S. Nadeem, S. Asghar, and A. M. Siddiqui, "Fluctuating flow of a third order fluid on a porous plate in a rotating medium," The International Journal of Non-Linear Mechanics, vol. 36, pp. 901916, 2001.

[23] T. Hayat and S. Abelman, "A numerical study of the influence of slip boundary condition on rotating flow," International Journal of Computational Fluid Dynamics, vol. 21, no. 1, pp. 21-27, 2007.

[24] S. Abelman, E. Momoniat, and T. Hayat, "Steady MHD flow of a third grade fluid in a rotating frame and porous space," Nonlinear Analysis: Real World Applications, vol. 10, no. 6, pp. 3322-3328, 2009.

[25] S. Abelman, E. Momoniat, and T. Hayat, "Couette flow of a third grade fluid with rotating frame and slip condition," Nonlinear Analysis: Real World Applications, vol. 10, no. 6, pp. 3329-3334, 2009.

[26] B. Sahoo, "Effects of slip on steady Bödewadt flow and heat transfer of an electrically conducting non-Newtonian fluid," Communications in Nonlinear Science and Numerical Simulation. In press.

[27] B. Sahoo, "Effects of partial slip, viscous dissipation and Joule heating on Von Kármán flow and heat transfer of an electrically conducting non-Newtonian fluid," Communications in Nonlinear Science and Numerical Simulation, vol. 14, no. 7, pp. 2982-2998, 2009. 


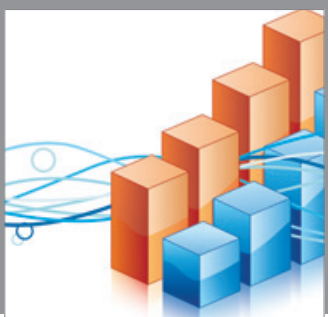

Advances in

Operations Research

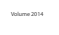

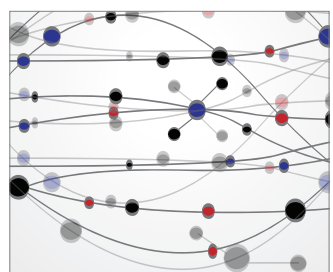

\section{The Scientific} World Journal
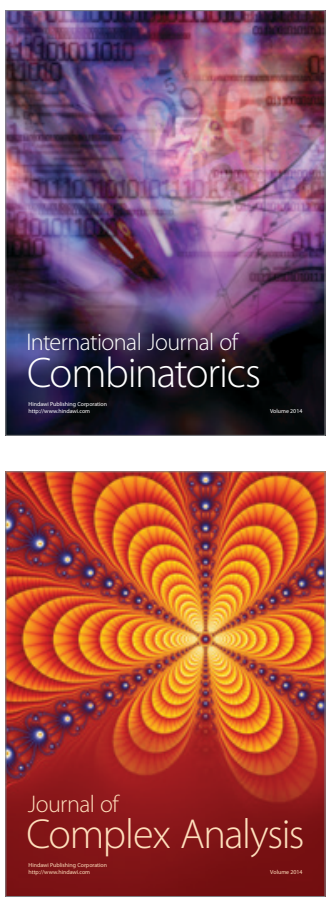

International Journal of

Mathematics and

Mathematical

Sciences
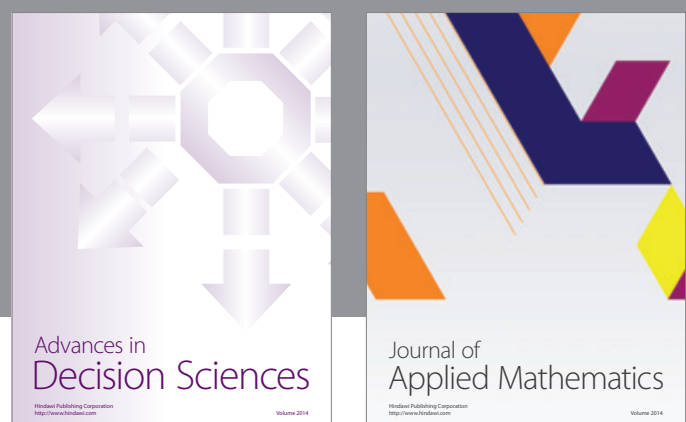

Journal of

Applied Mathematics
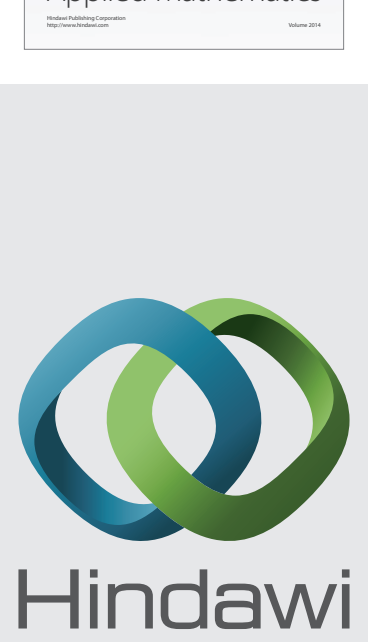

Submit your manuscripts at http://www.hindawi.com
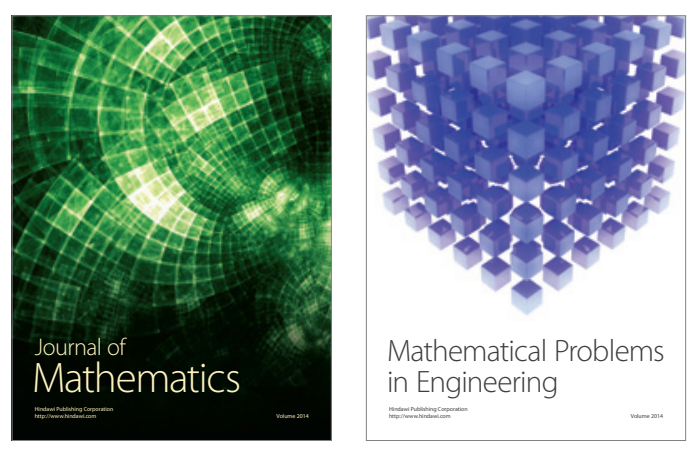

Mathematical Problems in Engineering
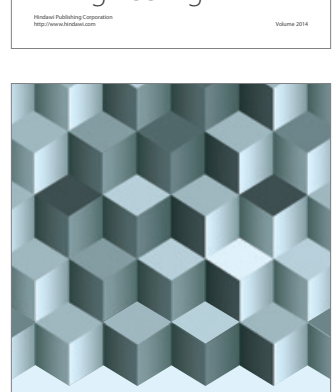

Journal of

Function Spaces
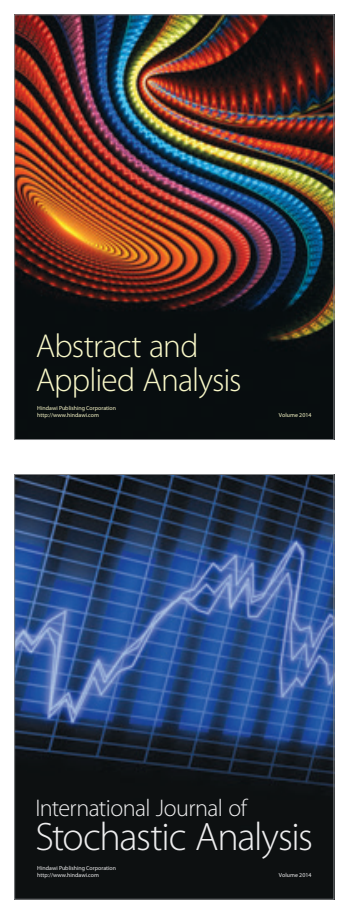

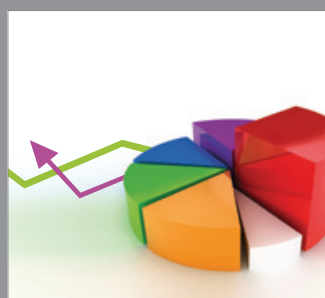

ournal of

Probability and Statistics

Promensencen
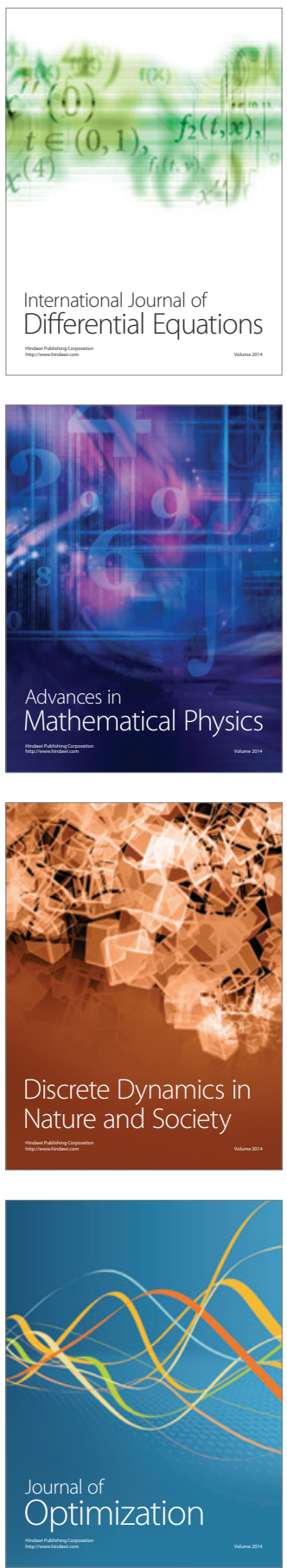